\title{
Utilização do método de Eletrorresistividade na determinação do capeamento e estruturas da subsuperfície na Mineração Lapa Vermelha - Confins - MG
}

Aranha, P. R. A ${ }^{1}$; Matta J. F. B. ${ }^{2 *}$ \& Santos M. A. ${ }^{3}$, Instituto de Ciências Exatas, Universidade Federal de Minas Gerais Copyright 2016, SBGf - Sociedade Brasileira de Geofísica

Este texto foi preparado para a apresentação no VII Simpósio Brasileiro de Geofísica, Ouro Preto, 25 a 27 de outubro de 2016. Seu conteúdo foi revisado pelo Comitê Técnico do VII SimBGf, mas não necessariamente representa a opinião da SBGf ou de seus associados. É proibida a reprodução total ou parcial deste material para propósitos comerciais sem prévia autorização da SBGf.

\section{Resumo}

A área pesquisada situa-se na Mineração Lapa Vermelha, pouco ao norte de Belo Horizonte, em ConfinsMG (Figura 1), e pertence a uma das regiões mais importantes em termos de paísagem cárstica carbonática, o carste de Lagoa Santa da Formação Sete Lagoas (Grupo Bambuí). Tal região possui um relevo superficial (exocarste), que evolui a partir da configuração de redes hídricas subterrâneas (endocarste) e de uma dinâmica intensa na interface rocha-solo (epicarste), onde é comum identificar paredões lineares, canyons, vales cegos e dolinas de abatimento.

O estudo desta área tem por objetivo investigar a variação horizontal de resistividade em várias profundidades e verificar a ocorrência de rocha calcária para exploração, para que a lavra possa ser desenvolvida de maneira adequada, segundo o padrão da empresa.

A metodologia escolhida foi baseada nos princípios geofísicos da eletrorresistividade, utilizando-se o método de caminhamento elétrico com arranjo dipolo-dipolo. A fim de tornar a pesquisa mais precisa foram realizados também furos de sondagem antes e após a realização dos caminhamentos elétricos para correlacioná-los aos perfis e obter maior consistência nos resultados. Os resultados obtidos foram satisfatórios, uma vez que mostraram, com boa precisão, as dimensões do capeamento de solo e as principais descontinuidades presentes no maciço.

\section{Abstract}

The studied area is located in Lapa Vermelha Mining, just north of Belo Horizonte, in Confins, Minas Gerais (Figure 1), and it belongs to one of the most important regions in karst carbonate landscape, the karst of Lagoa Santa in the Sete Lagoas formation(Bambuí Group). This region has hum surface relief (exocarste), which evolves from the configuration of groundwater networks (endokarst) and an intense dynamic in rock-soil interface (epicarste), where it is common to identify linear cliffs, canyons, blind valleys and collapse sinkholes.

This study aims to investigate the horizontal variation of resistivity at many depths and verify the occurrence of calcareous rock for exploration, so that the mining can be

developed in an appropriate way, according to the company standard.

The chosen methodology was based on the principles of geophysical eletric resistivity, using the eletrical traversal method with dipole-dipole arrangement. In order to make the search more precise, boreholes were done before and after the electrical traversal to correlate the profiles, therefore having more consistent results. The results were tolerable once they showed, with good accuracy, the soil capping size and the major descontinuities at the massive.

\section{Introdução}

A exploração de recursos minerais assumiu privilegiada importância em vários lugares e em Minas Gerais essas atividades abundantes têm contribuído imensamente para a riqueza nacional, com benefícios socioeconómicos associados e por isto se faz cada dia mais necessário o entendimento de métodos que auxiliam nestas explorações e por isto este estudo visa demostrar a eficácia do método geofísico de resistividade e sua importância.

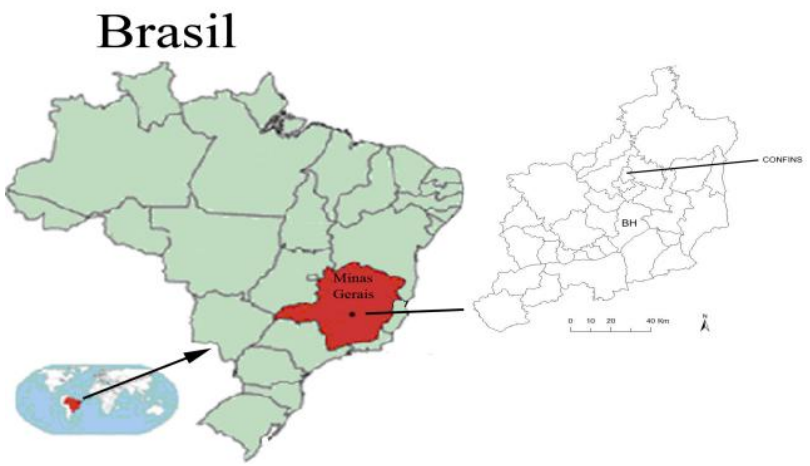

Figura 1: Localização da área estudada - Confins. 


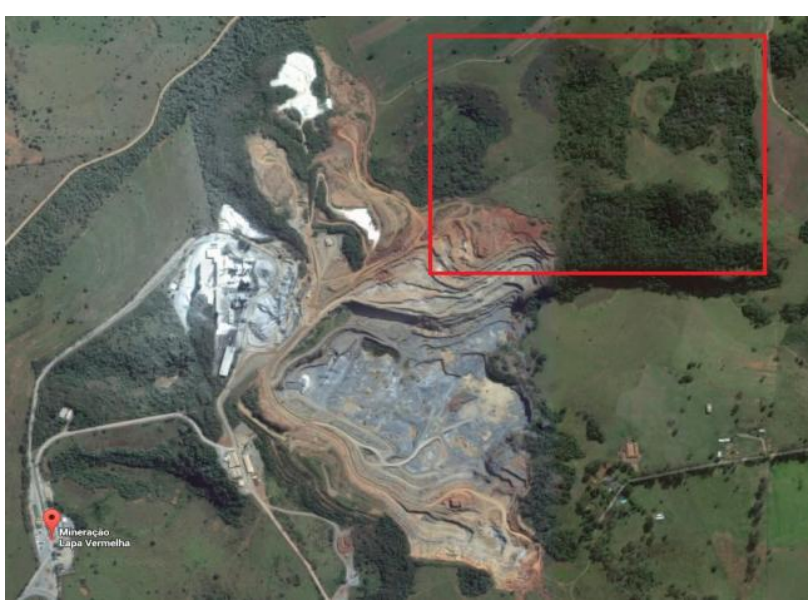

Figura 2: Imagem de satélite com a demarcação da área de estudo (quadro vermelho) na mina.

Na região estudada (Figuras 2 e 3) localizada na área da Mineração Lapa Vermelha, nas adjacências da metrópole Belo Horizonte, centro-sul do estado de Minas Gerais, é um importante exemplar brasileiro de ambiente cárstico desenvolvido em rochas carbonáticas. Nela foram executados Perfis Geofísicos: aquisição, processamento e interpretação de perfis de Eletrorresistividade (Caminhamento Elétrico) com o intuito de se determinar a presença de variações na resistividade aparente ocasionada pela variação direcional de alguma propriedade do solo, ou da rocha no substrato e determinar também a presença de descontinuidades falhas, fraturas, presença de carstificação (cavernas) e dolinas numa região calcária. E também delimitar a espessura do capeamento de solo para definir a forma e as características da lavra no local.

A utilização do método de eletrorresistividade foi aplicado nesta mina em função dos resultados deste método em outros estudos como, por exemplo, o trabalho apresentado no XVIII Congresso Brasileiro de Águas Subterrâneas por DUARTE et al. (2014) com levantamento de perfis feito na cidade de Nobres, MT onde menciona "a aplicação dos métodos de eletroresistividade na interpretação geofísico-geológica de terrenos cársticos, tem se revelado útil, considerando a possibilidade de identificação e dimensionamento de feições de dissolução tais como fraturas, vazios ou cavidades" e também o outro realizado pela Universidade Federal da Colombia por MUHAMMAD FAROOQ ET AL. (2012) na Coreia do Sul e publicado no Earth sciences research Journal onde ressalta que "as imagens de resistividade invertidas proporcionam uma visão clara de solos intemperizados, a distribuição de áreas fracas ou vazios cársticas".

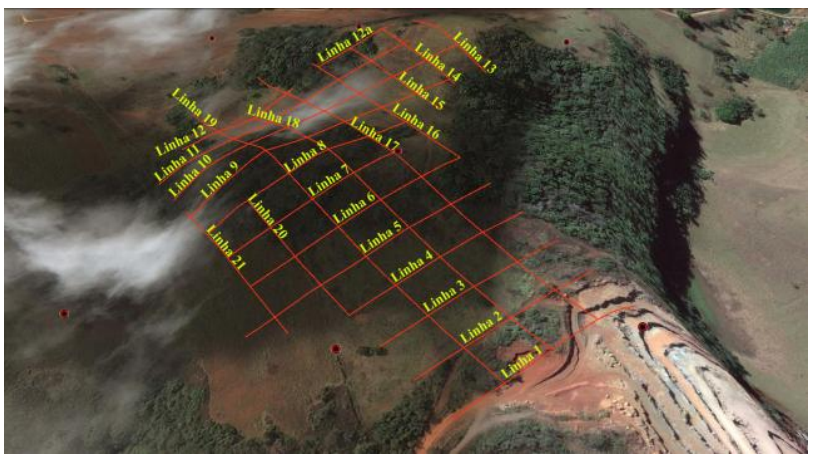

Figura 3: Imagem da região com a demarcação dos perfis realizados em vermelho.

\section{Metodologia}

Na obtenção de Dados foi escolhida a utilização do arranjo Dipolo-Dipolo, em função de suas características operacionais e dos resultados que proporciona. $\mathrm{O}$ arranjo escolhido utiliza quatro eletrodos, sendo dois de corrente e dois de potencial, que permite desenvolver a aquisição dos dados geoelétricos de forma contínua. O equipamento utilizado foi o SAS1000 da ABEM Terrameter, com cabos e conectores, eletrodos de aço inoxidável, com intervalo entre os eletrodos de $8 \mathrm{~m}, 64$ saídas no cabo, sendo o spread total em cada aquisição de $512 \mathrm{~m}$. Foi utilizado o modo roll along com cobertura de $50 \%$. A configuração da aquisição foi feita para a leitura de 18 níveis de investigação.

Obteve-se então através deste método informações de vários níveis de profundidade para diversas posições em subsuperfície. Para se ter um padrão de comparação, foi realizado também um perfil numa das bermas da mina (Fig. 3a), para ser o padrão de referência quanto às características da rocha e sua assinatura geoelétrica onde se tem a presença de rocha e uma descontinuidade, utilizou-se o mesmo equipamento e a mesma metodologia de aquisição dos dados. A seção geoelétrica, pode ser visualizada na figura 4b. Nesta seção observa-se na parte à esquerda e mais à direita valores altos de resistividade refletindo a presença de calcário maciço. Na parte central observam-se valores mais baixos de resistividade que foram correlacionados com presença de "fratura" na rocha, alterando os valores de resistividade. Esse lineamento também é visível na imagem da área (Fig. 2a). Desse modo, teve-se a referência de valores de resistividade para se interpretar os perfis que foram obtidos na área de interesse, região Norte. 


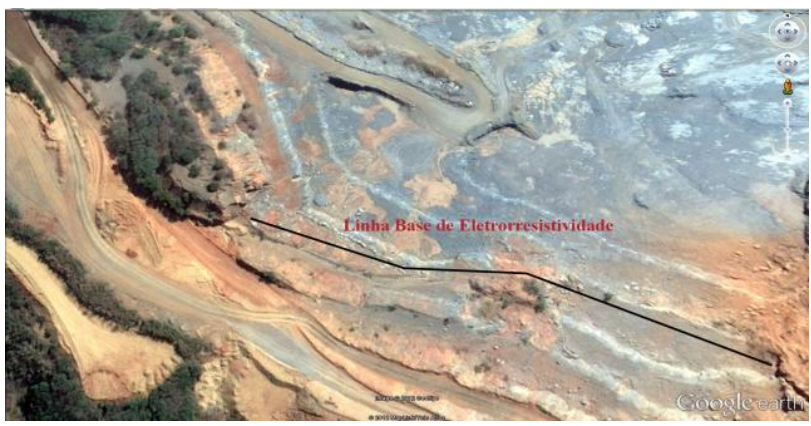

Figura 4a: Localização do perfil base na berma de calcário.

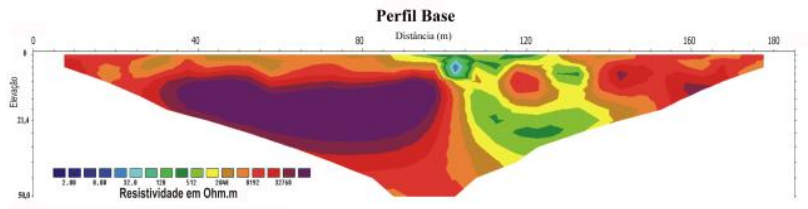

Figura 4b: Perfil base de eletrorresistividade

Os resultados de campo obtidos foram processados numa planilha eletrônica, utilizando a equação apropriada (Koefoed, 1979), para se calcular a resistividade aparente. Após esta etapa foi aplicada a inversão dos dados, isto é, encontrar um modelo da subsuperfície (em termos de resistividade) que apresentasse a mesma resposta, isto é, a mesma seção obtida no campo. Esses modelos de inversão a serem utilizados em programa adequado (Res2Dinv e Res3Dinv) foram plotados em forma de seções e "slices" de profundidade. As seções geradas são do tipo "pseudo-depth section", sendo então interpretados em função das variações das resistividades apresentadas, correlacionando-as com características geomorfo-geológicas da área.

\section{Resultados}

Os perfis 1 a 12 foram obtidos no sentido indicado pelo levantamento topográfico, esquerda-direita enquanto os perfis 13 a 21 foram adquiridos transversamente em relação aos primeiros perfis na direção sul-norte como pode ser observado analisando-se a figura 2. Foram criados dois mosaicos contendo as imagens geradas a partir dos dados adquiridos em campo. Todos os perfis estão com a mesma escala de cores e de unidade métrica.

O Perfil 1 (Figura 5) é seccionado pelos inícios dos perfis $16,17,18$ e 19 (conforme figura 3 ) e está paralelo ao perfil 2. Tem como ponto importante de ser observado que seu final, está localizado dentro da cava de exploração da mina, o que serviu como mais uma fonte de comparação das resistividades presentes no perfil. Nele observam-se valores baixos de resistividade na parte central, tendo caimento para a direita, possivelmente algum lineamento: fratura ou falha, ou mesmo uma zona de dissolução do calcário. Notam-se também algumas áreas isoladas com valores de resistividade mais altos, esses podem ser correlacionados com presença de matacões.

Para se interpretar melhor os dados coletados sobre a área em questão foram elaborados dois mosaicos (Figuras 11 e 12), um com os perfis na direção NW-SE colocados paralelamente entre si e outro com os perfis com direção perpendicular à primeira, SW-NE. Com base nestas figuras pode-se perceber a presença de lineamentos estruturais (fraturas/falhas) presentes na área, como também estimar as dimensões do maciço rochoso, isto é, suas continuidades laterais e em profundidade.

Antes de efetuar os caminhamentos um furo foi executado no cruzamento dos perfis 7 e 17, nas adjacências dos perfis 6 e 18 (figuras 7, 8, 9 e 10) e posteriormente às interpretações outro furo foi efetuado em torno dos $220 \mathrm{~m}$ do perfil 5 (Figura 6) a fim de correlacionar com os resultados obtidos e dar maior consistência a estes. Este furo investigativo, realizado ao término do trabalho, confirmou a existência de rocha por volta dos $12 \mathrm{~m}$ de profundidade conforme mostrado no perfil 5, estando de acordo com as interpretações dos perfis.

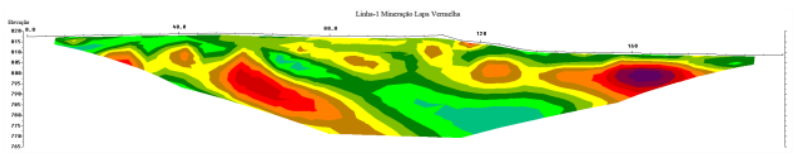

Figura 5: Perfil 1

O Perfil 5 (figura 6) é seccionado pelos perfis 17, 18, 19 e 20. A presença do lineamento que é observado nos perfis anteriores não está bem definida, e os valores baixos indicam a presença de solo, ou rocha bastante alterada. Na parte direita do perfil visualizam-se valores isolados mais altos de resistividade indicando a presença de rocha, sendo confirmado pelo furo investigativo realizado no fim do estudo.

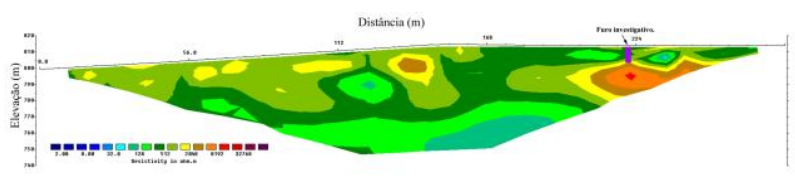

Figura 6: Perfil 5

Perfil 6 - apresenta valores de resistividade baixos como o perfil anterior, com uma diferença, que é uma região que indica a presença de matacões ou pequenas cavernas isoladas ao longo da seção. Estes matacões estariam envoltos em solo-rocha alterada, o que é corroborado pelos dados observados no furo de sondagem 11, realizado nas proximidades do perfil 


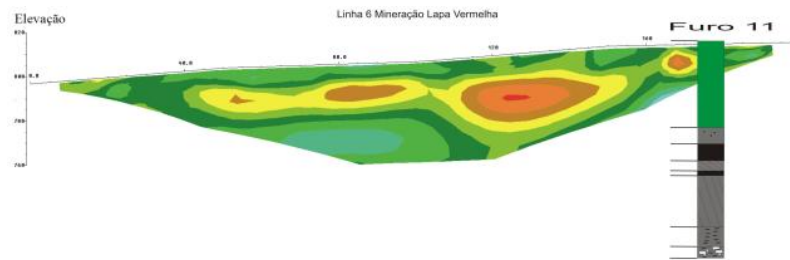

Figura 7: Perfil 6.

O Perfil 7 apresenta uma região com resistividades mais baixas que se estende de seu início até o final de extensão na parte superficial. Contudo na região entre 40 e $120 \mathrm{~m}$, na parte inferior observam-se valores elevados de resistividade, os quais podem ser correlacionados com presença de rocha. Esta interpretação encontra é consistente com os resultados observados no furo de sondagem 11 realizado nas proximidades do perfil.

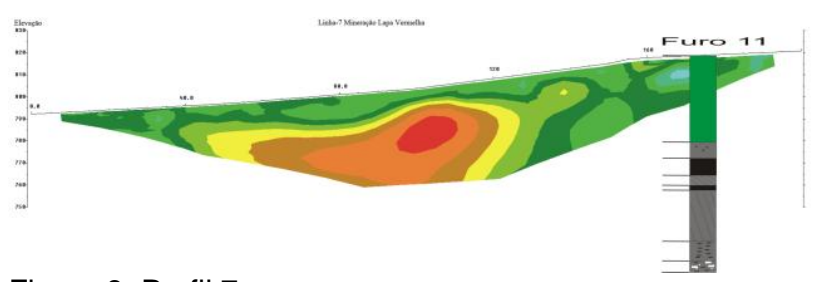

Figura 8: Perfil 7

O perfil 17 apresenta padrões de resistividade bem definidos ao longo de sua extensão. Em posições superficiais observa-se a presença de resistividades baixas que são encontradas em locais com a presença de solo. Mais abaixo tem-se a presença de valores altos de resistividade mostrando a presença de rocha, o que é corroborado pela análise das amostras recolhidas do furo de sondagem 11. Em geral o perfil é coerente com o apresentado nos pontos dos perfis de 1 a 12, que ele secciona. Mostrando uma camada de rocha em toda a sua extenção.

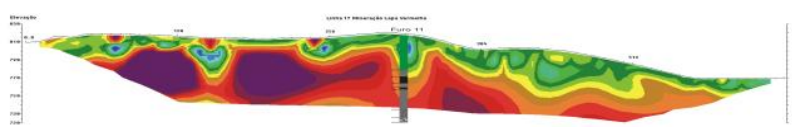

Figura 9: Perfil 17

Perfil 18 - Apresenta padrões de resistividade que variam ao longo de sua extensão. Do início do perfil até 300 metros em linha o perfil apresenta um padrão de resistividade de valores baixos, com uma pequena região de resistividades altas que podem caracterizar a presença de matacões. O restante do perfil apresenta um padrão bem diferente do encontrado em seu início, apresentando valores altos de resistividade. Esta interpretação é corroborada pelos resultados do furo de sondagem 11. Em geral o perfil também é coerente com o apresentado nos pontos dos perfis de 1 a 12 , que ele

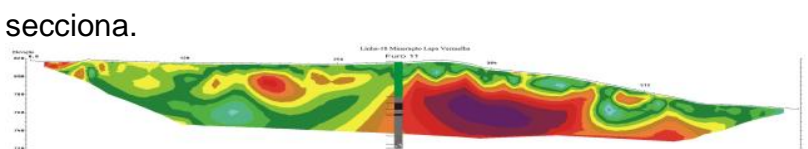

Figura10: Perfil 18

O mosaico 1 mostra o modelo de resistividade da subsuperfície, obtido com a inversão do caminhamento realizado com o arranjo dipolo-dipolo, para os perfis de 1 a 12. Nele observa-se também os perfis geológicos das sondagens furos 11 e 12 .

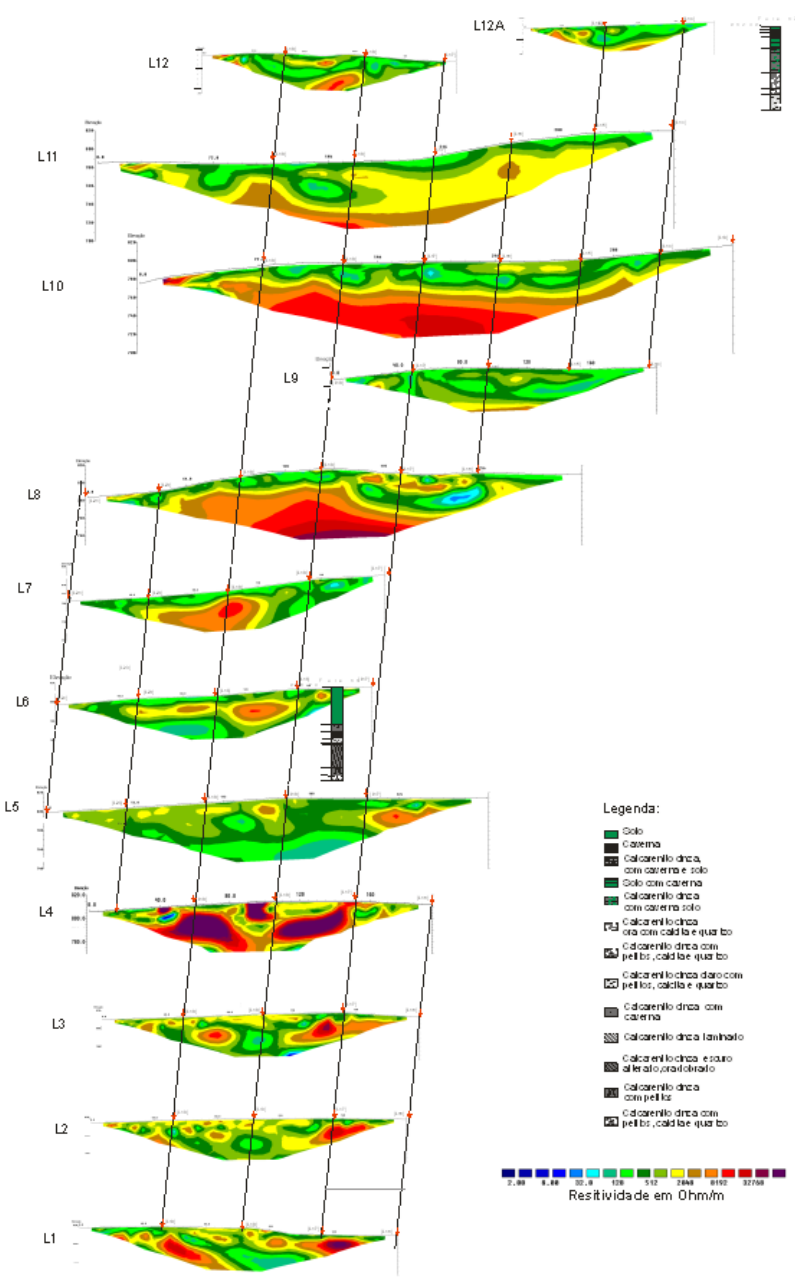

Figura 11: Mosaico 1

Já o mosaico 2 mostra o modelo de resistividade da subsuperfície, obtido com a inversão do caminhamento realizado com o arranjo dipolo-dipolo, para os perfis de 13 a 21 . E os perfis geológicos das sondagens furos $11 \mathrm{e}$ 12. 


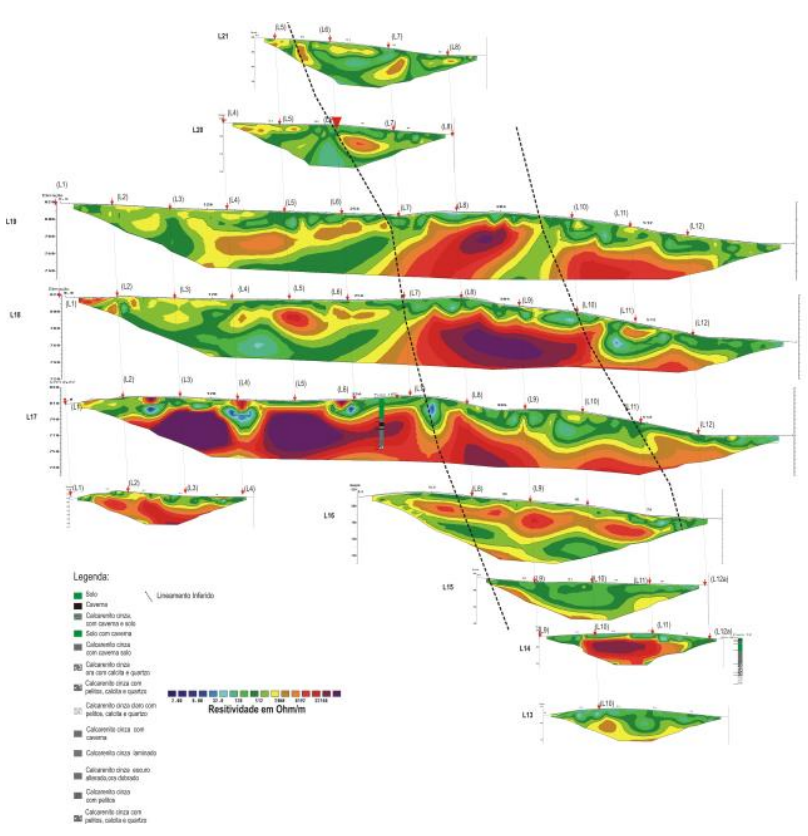

Figura 12: Mosaico 2

\section{Discussão e Conclusões}

O uso da dos métodos geofísicos tem se mostrado cada vez mais importantes para estudos investigativos de certas propriedades e aspectos da crosta terrestre. $\mathrm{O}$ presente estudo alcançou o objetivo e demostrou a eficácia do método de eletrorresistividade para identificação de estruturas e variações na subsuperfície dessas áreas.

Através do conjunto de perfis e dos mosaicos construídos pode-se perceber a presença de lineamentos estruturais (fraturas/falhas) presentes na área. Além disso, estimouse as dimensões do maciço rochoso, como podemos observar nos perfis $6,7,17$ e 18 e confirmado pelo furo de sondagem realizado no caminhamento 5 . Dessa forma foi evidenciado continuidades laterais e em profundidade, mostrando assim a ocorrência de rocha calcária para que a mina possa continuar em operação de forma adequada.

Notam-se também algumas áreas isoladas com valores de resistividade mais altos, esses podem ser correlacionados com presença de matacões.

A partir da correlação dos furos com os perfis de resistividade também foi possível constatar que os resultados obtidos demostram a eficácia das interpretações vistas, proporcionando um maior entendimento do comportamento deste método em ambientes cársticos, bem como trazendo maior entendimento e confiança para esta ferramenta de interpretação da subsuperfície.

Com isso pode-se concluir que os dados das sondagens elétricas indicaram uma boa coerência, corroborando as informações obtidas nas referências bibliográficas consultadas.

\section{Agradecimentos}

Ao Dpto. De Geofísica - IGC/UFMG, UFOP e SBGf.

\section{Referências}

KOEFOED, O. (1979) Geosounding Principles, 1 Resistivity Sounding Measuraments; Elsevier Science Publ. Co., Inc. New York.

BERBERT-BORN, M. Carste de Lagoa Santa - MG; SIGEP 15 - CPRM - MG

MENEZES R. M. X. (2009) Levantamento por Eletrorresistividade na Bacia de São José de Itaborai - Universidade Federal do Rio de Janeiro, Instituto de Geociências, Departamento de Geologia 2009

ZHU, J.; CURRENS, J. C. \& DINGER J. S. (2011) Challenges of using electrical resistivity method to locate karst conduits - A field case in the Inner Bluegrass Region, Kentucky. - Journal of Applied Geophysics

DUARTE, R. S. S.; MARQUES, M. V. F; MORAES, A. L. \& RIBEIRO, J. R. (2014) Aplicação do Método de Eletrorresistividade (CE) em área de risco Geotécnico em terreno Cárstico, Nobres - MT. - XVIII Congresso Brasileiro de águas subterrâneas.

HOOVER R. A. Geophysical Choices For Karst and mine investigations Harrisburg, Pennsylvania.- http://www.dot.state.fl.us/

FAROOQ M. (2012) Subsurface cavity detection in a karst environment using electrical resistivity (er): a a case study from yongweol-ri, South Korea - Earth Sciences Research Journal. 